See Article page XXX.

\section{Commentary: Left ventricular assist device infections: An ounce of prevention is worth more than a pound of cure}

\author{
Bryan A. Whitson, MD, PhD
}

Zhou and colleagues ${ }^{1}$ present their investigation into the influence of the first documented infection after left ventricular assist device (LVAD) implantation and the overall survival in a 5-year period utilizing the Society of Thoracic Surgeons Interagency Registry for Mechanically Assisted Circulatory Support. Although those of us in the field know this to be true anecdotally, Zhou and colleagues ${ }^{1}$ effectively utilized the national, administrative database in an effective fashion to demonstrate the influence that device-related infection has on limiting patient survival overall as well as a decrease in quality of life.

Inherent challenges of implantable LVADs are limited by the driveline and that interface between the external environment and the skin and the driveline. Additionally, the added risk of an implantable mechanical heart pump presents an ever-present notice for infection, which can be difficult if not impossible to treat, whether pump or drive line or endocarditis occurs. Tracking the true influence of these infectious challenges on patient survival and quality of life has been challenging. There are differences in definitions and lack of consensus regarding timing and progression of infection of whether they are sometimes considered 1 or multiple infections depending on the pathogen present, the location of infection, or the time course. To address this, these investigators utilize the best approach possible by looking at the timing and

\footnotetext{
From the Division of Cardiac Surgery, Department of Surgery, The Ohio State University Wexner Medical Center, Columbus, Ohio.

Disclosures: The author reported no conflicts of interest.

The Journal policy requires editors and reviewers to disclose conflicts of interest and to decline handling or reviewing manuscripts for which they may have a conflict of interest. The editors and reviewers of this article have no conflicts of interest.

Received for publication Dec 8, 2021; revisions received Dec 8, 2021; accepted for publication Dec 9, 2021.

Address for reprints: Bryan A. Whitson, MD, PhD, Division of Cardiac Surgery, Department of Surgery, Ohio State University Wexner Medical Center, N-816

Doan Hall, 410 W 10th Ave, Columbus, OH 43210 (E-mail: bryan.whitson@ osumc.edu).

J Thorac Cardiovasc Surg 2021; $\mathbf{\square}: 1-2$

$0022-5223 / \$ 36.00$

Copyright (C) 2021 Published by Elsevier Inc. on behalf of The American Association for Thoracic Surgery

https://doi.org/10.1016/j.jtcvs.2021.12.019
}

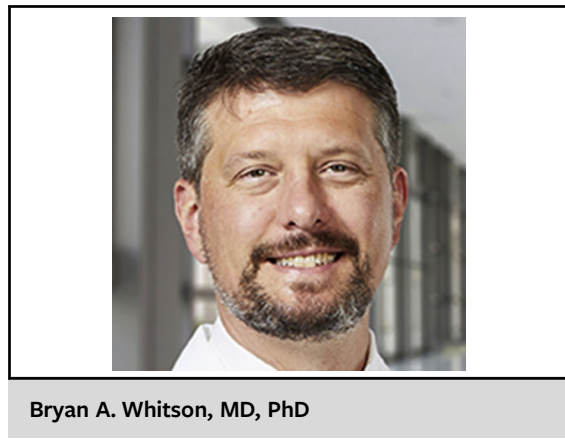

\section{CENTRAL MESSAGE \\ Meticulous attention to pre- venting infectious complications is critical to overall survival and quality of life after left ventricular assist device implantation.}

influence of the first infection that has been documented. Although there are some inherent limitations of this type of work and the authors clearly identify them (eg, inability to identify a specific organism or progression of some infections or the nature of known deficiencies of administrative data sets), this analysis does provide an effective evaluation of LVAD-related infectious complications that are based on prospectively gathered data with common, agreed-upon definitions and the robust nature of the Society of Thoracic Surgeons Interagency Registry for Mechanically Assisted Circulatory Support collection infrastructure.

Two points are of critical importance. The first is the demonstration that the identification of an early, nonLVAD infection is a significant precursor to subsequent mortality. The second is the surprising observation that approximately half of implanted patients developed an infection during the study period.

In the current era, after the heart transplant allocation changes of 2018, these results are incredibly poignant from the perspective that LVADs are potentially more often being utilized as destination therapy instead of bridges to heart transplantation. That being the case, the role of the timing of that first infection, its location, and the subsequent influence on survival and quality of life is even more pronounced because there may not be transplant options readily available for these patients.

With this observation and correlation of timing of infection and the overall high incidence in these patients 
with LVADs, the highly important role of education can be the takeaway. Education with the patient and his or her family coupled with an aggressive programmatic approach to driveline site management and infection prevention and aggressive treatment are paramount to keeping these patients free from infection and subsequently improving not only their quality of life but overall survival. Zhou and colleagues ${ }^{1}$ are to be commended for their influential work.

\section{Reference}

1. Zhou S, Yang G, Zhang M, Pienta M, Chenoweth CE, Pagani FD, et al. Mortality following durable left ventricular assist device implantation by timing and type of first infection. J Thorac Cardiovasc Surg. 2022. XX:XX-X. 